\title{
Revision to CDC's Zika Travel Notices: Minimal Likelihood for Mosquito-Borne Zika Virus Transmission at Elevations Above 2,000 Meters
}

\author{
Martin Cetron, $\mathrm{MD}^{1}$
}

On March 11, 2016, this report was posted as an MMWR Early Release on the MMWR website (http://www.cdc.gov/mmwr).

Since May 2015, when Zika virus, a flavivirus transmitted primarily by Aedes aegypti mosquitoes, was reported in Brazil, the virus has rapidly spread across the Region of the Americas and the Caribbean. The association between maternal Zika virus infection and adverse fetal and reproductive outcomes, including microcephaly, prompted CDC to issue a Level 2 alert travel notice* for the 37 countries and U.S. territories (at the national and territorial level) that have reported recent Zika virus transmission as of March 11, 2016. In addition to mosquito bite precautions for all travelers, CDC advises that pregnant women postpone travel to affected countries and U.S. territories. Within a nation's borders, ecologic characteristics, which determine the distribution of mosquito vectors, can vary considerably. CDC conducted a spatial analysis, focusing on the probability of occurrence of Ae. aegypti, to support the demarcation for subnational travel alerts. Based on results of this analysis, travel that is limited to elevations higher than 2,000 $\mathrm{m}(6,562 \mathrm{ft})$ above sea level is considered to have minimal (approximately 1\%) likelihood for mosquitoborne Zika virus transmission, even within countries reporting active transmission. Women who are pregnant should avoid travel to elevations $<2,000 \mathrm{~m}$ in countries with active Zika virus transmission.

Zika virus is a flavivirus primarily transmitted by Aedes species mosquitoes (1). In May 2015, the Pan American Health Organization (PAHO) issued an alert regarding the first confirmed Zika virus infections in Brazil (2). Currently, outbreaks of Zika virus disease are occurring in many countries and U.S. territories, and as of March 11, 2016, CDC had issued 37 Level 2 travel notices for areas with ongoing Zika virus transmission. ${ }^{\dagger}$ Currently, when laboratory-confirmed local Zika virus transmission is first reported, travel notices are issued for the entire country or U.S. territory. Establishing more precisely defined areas of Zika virus risk in a country or U.S. territory

\footnotetext{
${ }^{*} \mathrm{CDC}$ provides updated travel information on areas with ongoing Zika virus transmission. http://wwwnc.cdc.gov/travel/notices.

$\dagger$ American Samoa, Aruba, Barbados, Bolivia, Bonaire, Brazil, Cape Verde, Colombia, Costa Rica, Curacao, Dominican Republic, Ecuador, El Salvador, French Guiana, Guadeloupe, Guatemala, Guyana, Haiti, Honduras, Jamaica, Marshall Islands, Martinique, Mexico, New Caledonia, Nicaragua, Panama, Paraguay, Puerto Rico, Saint Martin, Saint Vincent and Grenadines, Samoa, Sint Maarten, Suriname, Tonga, Trinidad and Tobago, U.S. Virgin Islands, and Venezuela.
}

is complicated by incomplete surveillance data on the disease and the presence of the mosquito vector.

In an effort to develop more precise guidance for travelers, CDC evaluated whether subnational travel notices could be based on an ecologic indicator of the probable absence of the predominant Zika virus mosquito vector, Ae. aegypti. Within a nation's borders, ecologic factors, such as temperature, precipitation, vegetation, and human population density, that define suitable habitats for Aedes species vary. Where habitat is unsuitable, the mosquito vector is likely to be absent, and risk for mosquito-borne Zika virus transmission is likely to be negligible.

The first step in developing subnational travel notices required identification of a single, easily quantifiable ecologic variable that could be used as a substitute for the likely absence of Ae. aegypti. Of the many ecologic factors affecting habitat suitability and Ae. aegypti survival as a vector for Zika virus, temperature has been the most frequently investigated and rigorously quantified (3); however, temperature varies widely and is difficult to predict locally and over the long term. Historically, elevation has served as a reasonable proxy for temperature. Because it is static and relatively easy to measure (4), elevation was selected for further investigation. Previous reports from various global regions suggest that $A$ e. aegypti is present, but rare, between elevations of 1,700-2,100 m (5,6). Therefore, this analysis was restricted to countries and U.S. territories that have 1) ongoing Zika virus transmission and 2) areas with high elevations (starting at $>1,500 \mathrm{~m}$ ). Sixteen countries, including Bolivia, Brazil, Colombia, Costa Rica, Dominican Republic, Ecuador, El Salvador, Guatemala, Guyana, Haiti, Honduras, Jamaica, Mexico, Nicaragua, Panama, and Venezuela have areas which fit these criteria. ${ }^{\$}$ No U.S. territories had elevations at that level.

Spatial analyses were conducted using multiple data sets: global data on predicted probabilities of the presence of Ae aegypti based on 20,000 observed occurrences during 1960-2014 (7); remotely sensed data on human population density (8); global geographic data on human dengue cases

\footnotetext{
${ }^{\$} \mathrm{CDC}$ provides updated travel notice maps for areas with ongoing Zika virus transmission, including Bolivia, Brazil, Colombia, Costa Rica, Dominican Republic, Ecuador, El Salvador, Guatemala, Guyana, Haiti, Honduras, Jamaica, Mexico, Nicaragua, Panama, and Venezuela. http://wwwnc.cdc.gov/travel/page/ zika-travel-information.
} 
during 1960-2012 (9); and a digital elevation model (10); zonal statistics were used to relate the data sets. Within each of the 16 countries, the area of land suitable for Ae. aegypti, and the human population counts within each area were quantified. The quantification was done in 100-m elevation segments for elevations between $0 \mathrm{~m}$ and 2,500 m. Across all 16 countries, at elevations $>2,000 \mathrm{~m}, A e$. aegypti was predicted to be largely absent. Because of sparse current geographic data on Zika virus cases, cases of dengue, another vector-borne viral disease spread primarily by $A$ e aegypti, were examined as a proxy for Zika cases. Only $1.1 \%(28 / 2,682)$ of dengue cases in the global data set (9) were reported to have occurred at elevations $>2,000 \mathrm{~m}$ in the 16 countries.

A CDC Zika virus travel notice is currently applied to an entire country or U.S. territory when transmission is confirmed by a local public health authority. However, Ae. aegypti might not be uniformly present because of differences in ecologic suitability. Recent advances in scientific modeling have allowed for more precision in geospatial analyses. CDC applied these approaches to previously published and rigorously evaluated data to determine if more precise guidance to travelers and persons living in affected regions could be established. The results from the spatial analyses of 16 countries with ongoing Zika virus transmission and elevation points $>1,500 \mathrm{~m}$ indicate that $A$ e. aegypti is unlikely to be found at elevations $>2,000 \mathrm{~m}$ because of unsuitable ecologic factors, including but not limited to, low temperatures. Consequently, at elevations above 2,000 $\mathrm{m}$, the risk for mosquito-borne exposure to Zika virus is considered to be minimal. These findings support revising the Zika travel notice to reflect enhanced geographic precision regarding the likelihood of Zika virus presence at certain elevations.

With this revision, CDC recommends that women who are pregnant should postpone travel to areas that are at elevations $<2,000 \mathrm{~m}$ above sea level in countries and U.S. territories with ongoing Zika virus transmission. Because Zika virus is primarily spread by mosquitoes, CDC recommends that travelers protect themselves from mosquito bites. Travel that is entirely limited to elevations $>2,000 \mathrm{~m}$ is considered to pose minimal likelihood for mosquito-borne Zika virus transmission. ${ }^{* *}$ As additional geographic data specific to Zika virus cases in relation to elevation become available, these recommendations will be reviewed and revised as needed.

\footnotetext{
http://wwwnc.cdc.gov/travel/page/avoid-bug-bites.

** The low oxygen levels found at high elevations can cause problems for travelers who are going to elevations above $2,400 \mathrm{~m}(8,000 \mathrm{ft})$. The best way to prevent altitude illness is to ascend slowly and take time to get used to the lower oxygen levels. Pregnant women should avoid strenuous activities at high elevations, and some doctors recommend that pregnant women not spend the night at altitudes above 3,650 m (12,000 ft). Pregnant women should also consider whether they will have access to medical care at a high-elevation destination.
}

${ }^{1}$ Division of Global Migration and Quarantine, National Center for Emerging and Zoonotic Infectious Diseases, CDC.

Corresponding author: Martin Cetron, MCetron@cdc.gov, 770-488-7100.

\section{References}

1. Chouin-Carneiro T, Vega-Rua A, Vazeille M, et al. Differential susceptibilities of Aedes aegypti and Aedes albopictus from the Americas to Zika virus. PLoS Negl Trop Dis 2016;10:e0004543. http://dx.doi. org/10.1371/journal.pntd.0004543

2. Pan American Health Organization. Epidemiological alert: Zika virus infection. 2015 May 7. Washington, DC: Pan American Health Organization, World Health Organization; 2015. http://www.paho.org/hq/index. php?option=com_docman\&task=doc_view\&Itemid=270\&gid=30075

3. Brady OJ, Golding N, Pigott DM, et al. Global temperature constraints on Aedes aegypti and Ae. albopictus persistence and competence for dengue virus transmission. Parasit Vectors 2014;7:338. http://dx.doi. org/10.1186/1756-3305-7-338

4. Garnham PC. Malaria epidemics at exceptionally high altitudes. BMJ 1945;2:45-7. http://dx.doi.org/10.1136/bmj.2.4410.45

5. Lozano-Fuentes S, Hayden MH, Welsh-Rodriguez C, et al. The dengue virus mosquito vector Aedes aegypti at high elevation in Mexico. Am J Trop Med Hyg 2012;87:902-9. http://dx.doi.org/10.4269/ ajtmh.2012.12-0244

6. Dhimal M, Gautam I, Joshi HD, O’Hara RB, Ahrens B, Kuch U. Risk factors for the presence of chikungunya and dengue vectors (Aedes aegypti and Aedes albopictus), their altitudinal distribution and climatic determinants of their abundance in central Nepal. PLoS Negl Trop Dis 2015;9:e0003545. http://dx.doi.org/10.1371/journal.pntd.0003545

7. Kraemer MU, Sinka ME, Duda KA, et al. The global distribution of the arbovirus vectors Aedes aegypti and Ae. albopictus. eLife 2015;4:e08347. http://dx.doi.org/10.7554/eLife.08347

8. LandScan. 2014. High resolution global population data set copyrighted by UT-Battelle, LLC, operator of Oak Ridge National Laboratory under contract no. DE-AC05-00OR22725 [dataset]. 2014. http://web.ornl. gov/sci/landscan/

9. Messina JP, Brady OJ, Pigott DM, Brownstein JS, Hoen AG, Hay SI. A global compendium of human dengue virus occurrence. Sci Data 2014;1:140004. http://dx.doi.org/10.1038/sdata.2014.4

10. Danielson JJ, Gesch DB. Global multi-resolution terrain elevation data 2010 (GMTED2010): US Geological Survey Open-File Report. Washington, DC: US Department of the Interior, US Geological Survey; 2011. http://pubs.usgs.gov/of/2011/1073/pdf/of2011-1073.pdf 